\title{
Bound state solutions of sublinear Schrödinger equations with lack of compactness
}

\author{
Anouar Bahrouni ${ }^{1}$ • Hichem Ounaies ${ }^{1}$. \\ Vicenţiu D. Rădulescu ${ }^{2,3}$
}

Received: 10 April 2018 / Accepted: 26 April 2018 / Published online: 5 May 2018

(C) The Author(s) 2018

Abstract We consider the following nonlinear Schrödinger equation

$$
-\Delta u+V(x) u=a(x)|u|^{q-1} u+f(x), \quad x \in \mathbb{R}^{N},
$$

where $V$ is a non-symmetric bounded potential, $a$ is an indefinite weight, $0<q<1$ and $f \neq 0$ is a nonnegative perturbation such that $f \in L^{2}\left(\mathbb{R}^{N}\right) \cap L^{\frac{2 N}{N+2}}\left(\mathbb{R}^{N}\right)$. Using variational methods, we prove the existence of two solutions with negative and positive energies, one of these solutions being nonnegative.

Keywords Nonlinear Schrödinger equation - Sublinear equation · Variational method . Multiple solutions

Mathematics Subject Classification $35 \mathrm{~J} 60 \cdot 35 \mathrm{~J} 91 \cdot 58 \mathrm{E} 30$

\section{Introduction}

The Schrödinger equation is central in quantum mechanics and it plays the role of Newton's laws and conservation of energy in classical mechanics, that is, it predicts the future behaviour

Vicenţiu D. Rădulescu

vicentiu.radulescu@imar.ro

Anouar Bahrouni

bahrounianouar@yahoo.fr

Hichem Ounaies

hichem.ounaies@fsm.rnu.tn

1 Mathematics Department, University of Monastir, Faculty of Sciences, 5019 Monastir, Tunisia

2 Faculty of Applied Mathematics, AGH University of Science and Technology, al. Mickiewicza 30, 30-059 Kraków, Poland

3 Institute of Mathematics "Simion Stoilow" of the Romanian Academy, P.O. Box 1-764, 014700 Bucharest, Romania 
of a dynamical system. It is striking to point out that talking about his celebrating equation, Erwin Schrödinger said: "I don't like it, and I'm sorry I ever had anything to do with it". The linear Schrödinger equation is a central tool of quantum mechanics, which provides a thorough description of a particle in a non-relativistic setting. Schrödinger's linear equation is

$$
\Delta \psi+\frac{8 \pi^{2} m}{\hbar^{2}}(E-V(x)) \psi=0,
$$

where $\psi$ is the Schrödinger wave function, $m$ is the mass of the particle, $\hbar$ denotes Planck's renormalized constant, $E$ is the energy, and $V$ stands for the potential energy.

Schrödinger also established the classical derivation of his equation, based upon the analogy between mechanics and optics, and closer to de Broglie's ideas. He developed a perturbation method, inspired by the work of Lord Rayleigh in acoustics, proved the equivalence between his wave mechanics and Heisenberg's matrix, and introduced the time dependent Schrödinger's equation

$$
i \hbar \psi_{t}=-\frac{\hbar^{2}}{2 m} \Delta \psi+V(x) \psi-\gamma|\psi|^{p-1} \psi \quad x \in \mathbb{R}^{N}(N \geq 2),
$$

where $p<2 N /(N-2)$ if $N \geq 3$ and $p<+\infty$ if $N=2$.

In physical problems, a cubic nonlinearity corresponding to $p=3$ in equation (1.1) is common; in this case problem (1.1) is called the Gross-Pitaevskii equation. In the study of equation (1.1), Floer and Weinstein [26] and $\mathrm{Oh}$ [36] supposed that the potential $V$ is bounded and possesses a non-degenerate critical point at $x=0$. More precisely, it is assumed that $V$ belongs to the class $\left(V_{a}\right)$ (for some real number $a$ ) introduced in Kato [31]. Taking $\gamma>0$ and $\hbar>0$ sufficiently small and using a Lyapunov-Schmidt type reduction, Oh [36] proved the existence of bound state solutions of problem (1.1), that is, a solution of the form

$$
\psi(x, t)=e^{-i E t / \hbar} u(x) .
$$

Using the Ansatz (1.2), we reduce the nonlinear Schrödinger equation (1.1) to the semilinear elliptic equation

$$
-\frac{\hbar^{2}}{2 m} \Delta u+(V(x)-E) u=|u|^{p-1} u .
$$

The change of variable $y=\hbar^{-1} x$ (and replacing $y$ by $x$ ) yields

$$
-\Delta u+2 m\left(V_{\hbar}(x)-E\right) u=|u|^{p-1} u \quad x \in \mathbb{R}^{N},
$$

where $V_{\hbar}(x)=V(\hbar x)$.

Let us also recall that in his 1928 pioneering paper, Gamow [27] proved the tunneling effect, which lead to the construction of the electronic microscope and the correct study of the alpha radioactivity. The notion of "solution" used by him was not explicitly mentioned in the paper but it is coherent with the notion of weak solution introduced several years later by other authors such as Leray, Sobolev and Schwartz. Most of the study developed by Gamow was concerned with the bound states $\psi(x, t)$ defined in (1.2), where $u$ solves the stationary equation

$$
-\Delta u+V(x) u=\lambda u \text { in } \mathbb{R}^{N},
$$

for a given potential $V(x)$. Gamow was particularly interested in the Coulomb potential but he also proposed to replace the resulting potential by a simple potential that keeps the main 
properties of the original one. In this way, if $\Omega$ is a subdomain of $\mathbb{R}^{N}$, Gamow proposed to use the finite well potential

$$
V_{q, \Omega}(x)=\left\{\begin{array}{ll}
V(x) & \text { if } x \in \Omega \\
q & \text { if } x \in \mathbb{R}^{N} \backslash \Omega
\end{array} \quad \text { for some } q \in \mathbb{R} .\right.
$$

It seems that the first reference dealing with the limit case, the so-called infinite well potential,

$$
V_{\infty}\left(x ; R, V_{0}\right)=\left\{\begin{array}{l}
V_{0} \text { if } x \in \Omega \\
+\infty \text { if } x \in \mathbb{R}^{N} \backslash \Omega
\end{array} \text { for some } V_{0} \in \mathbb{R},\right.
$$

was the book by the 1977 Nobel Prize Mott [35]. The more singular case in which $V_{0}$ is the Dirac mass $\delta_{0}$ is related with the so-called Quantum Dots, see Joglekar [29]. In contrast with classical mechanics, in quantum mechanics the incertitude appears (the Heisenberg principle). For instance, for a free particle (i.e. with $V(x) \equiv 0$ ), in nonrelativistic quantum mechanics, if the wave function $\psi(\cdot, t)$ at time $t=0$ vanishes outside some compact region $\bar{\Omega}$ then at an arbitrarily short time later the wave function is nonzero arbitrarily far away from the original region $\bar{\Omega}$. Thus, the wave function instantaneously spreads to infinity and the probability of finding the particle arbitrarily far away from the initial region is nonzero for all $t>0$. We refer to Díaz [25] for more details.

\section{The main results}

In this paper, we consider Schrödinger equations with sublinear nonlinearity and nonsymmetric potentials, which are affected by a nonnegative perturbation. We are interested in the multiplicity of solutions and we establish several sufficient conditions for the existence of two solutions.

We point out that sublinear problems on the whole space do not have necessarily a solution. In fact, the existence of solutions is in relationship not only with the nonlinearity but also with the behaviour of a certain potential. Brezis and Kamin [18] pointed out a striking phenomenon, which asserts that a sublinear problem on the whole space has a solution if and only if a linear equation depending only on the potential has a solution. They considered the nonlinear problem

$$
-\Delta u=\rho(x) u^{p}, \quad x \in \mathbb{R}^{n}(n \geq 3),
$$

with $0<p<1, \rho \in L_{\mathrm{loc}}^{\infty}\left(\mathbb{R}^{n}\right) \backslash\{0\}, \rho \geq 0$. Brezis and Kamin [18] proved that the nonlinear problem (2.1) has a bounded positive solution if and only if the linear equation

$$
-\Delta u=\rho(x), \quad x \in \mathbb{R}^{n}
$$

has a bounded solution. Their analysis showed that such a solution exists for potentials like

$$
\rho(x)=\frac{1}{1+|x|^{\alpha}} \quad \text { or } \quad \rho(x)=\frac{1}{\left(1+|x|^{2}\right)|\log (2+|x|)|^{\alpha}} \quad(\alpha>2),
$$

while no solution exists if

$$
\rho(x)=\frac{1}{1+|x|^{\alpha}} \quad \text { with } \alpha \leq 2 .
$$

Consider the following class of sublinear Schrödinger equations

$$
-\Delta u+V(x) u=a(x)|u|^{q-1} u+f(x), \quad x \in \mathbb{R}^{N},
$$


where

$$
N \geq 3,0<q<1, f \geq 0, f \neq 0 \text { and } f \in L^{2}\left(\mathbb{R}^{N}\right) \cap L^{\frac{2 N}{N+2}}\left(\mathbb{R}^{N}\right) .
$$

Equation (2.2) arises in the study of solitary waves in nonlinear equations of the KleinGordon or Schrödinger type.

We look for the existence of two solutions for problem (2.2), where the potential $V(x)$ and the weight $a(x)$ are indefinite, that is, they are sign-changing functions in $\mathbb{R}^{N}$. Such problems, with indefinite linear and nonlinear terms, present challenging mathematical difficulties.

Equation (2.2) can be considered as a perturbation of the homogeneous problem

$$
-\Delta u+V(x) u=a(x)|u|^{q-1} u, \quad u \in H^{1}\left(\mathbb{R}^{N}\right) \cap L^{q+1}\left(\mathbb{R}^{N}\right) .
$$

Equation (2.3) has been extensively studied in the last few decades, see [6-8, 18,20,21,38]. In 2008, using the Nehari manifold method, Chabrowski and Costa [21] proved the existence of two solutions of problem (2.3), where both $a(x)$ and $V(x)$ change sign in $\mathbb{R}^{N}$. In [7], Bahrouni et al. improved this result and established the existence of infinitely many solutions of problem (2.3) when $V(x)$ and $a(x)$ change sign.

In the case of bounded domains and under Dirichlet boundary conditions, there is a large literature on existence and a multiplicity of solutions for (2.2), see [3,5,17, 19, 28, 30,32,34] and the references therein. In a pioneering paper, Ahmad, Lazer and Paul [3] considered the resonant problem

$$
\begin{cases}-\Delta u-\lambda_{k} u=g(x, u) & \text { in } \Omega \\ u=0 & \text { on } \partial \Omega,\end{cases}
$$

where $\lambda_{k}$ denotes the $k$ th eigenvalue of the Laplace operator. They proved that a sufficient condition for the existence of a solution is

$$
\lim _{\|u\| \rightarrow+\infty, u \in E_{0}} \int_{\Omega} G(x, u(x)) d x=\mp \infty
$$

where $G(x, s)=\int_{0}^{s} g(x, t) d t$ and $E_{0}=\operatorname{Ker}\left(-\Delta-\lambda_{k}\right)$.

Brown [19] proved the existence of two solutions of problem (2.2) when $V=f=0$ and $a(x)$ changes sign.

In the case of unbounded domains, many authors have studied the existence of solutions of problem (2.2) with superlinear subcritical nonlinearity $\left(1<q<2^{*}=\frac{2 N}{N-2}\right)$, see $[1,2,4,33]$. For instance, $\mathrm{Li}$ and $\mathrm{Wu}[33]$ treated problem (2.2) where $f=\mu h, V=\lambda$ and $a(x) \in(0,1)$. The authors proved the existence of positive numbers $\epsilon>0$ and $\lambda_{0}, \mu>0$ such that for any $\lambda>\lambda_{0}$ and $\mu \lambda^{\frac{N}{4}-\frac{p-1}{p-2}}<\epsilon$, problem (2.2) admits multiple positive solutions.

For the sublinear case and especially for the whole space $\mathbb{R}^{N}$, to our best knowledge, few results are known. We can for example quote the papers [5,14-16]. For instance, Benrhouma [14] proved the existence of at least three solutions of problem (2.2), provided that $a(x)<0$ and $V$ changes sign. As far as we know, the only existence result for problem (2.2) where both $a$ and $V$ change sign in $\mathbb{R}^{N}$, is obtained by Tehrani [37]. He considered the equation

$$
-\Delta u+V(x) u=a(x) g(u)+f,
$$

under the following assumptions:

$\left(G_{1}\right) \quad g \in C(\mathbb{R}, \mathbb{R}), g(s) s \geq 0$ for all $s \in \mathbb{R} ;$

$\left(G_{2}\right) \lim _{|s| \rightarrow+\infty} \frac{g(s)}{|s|^{q-1} s}=1$, for some $0<q<1$; 
$\left(G_{3}\right) \exists A>0, \quad|g(s)| \leq A|s|^{q}$ for all $s \in \mathbb{R}$;

$\left(V_{2}\right) V \in L^{\infty}\left(\mathbb{R}^{N}\right)$ and $\liminf |x| \rightarrow+\infty V(x)=v_{\infty}>0$;

(H) $\int_{\mathbb{R}^{N}}\left[|\nabla \phi|^{2}+V(x) \phi^{2}\right] d x<0$ for some $\phi \in C_{c}^{\infty}\left(\mathbb{R}^{N}\right)$;

$\left(V_{3}\right) \quad 0 \in \sigma(-\Delta+V)$;

$\left(A_{0}^{\prime}\right) \quad a \in L^{\infty}\left(\mathbb{R}^{N}\right)$ and $\lim \sup _{|x| \rightarrow+\infty} a(x)=a_{\infty}<0$;

$\left(F_{1}\right) f \in L^{2}\left(\mathbb{R}^{N}\right)$;

$\left(N^{ \pm}\right) \int_{\mathbb{R}^{N}} a(x)|\varphi|^{q+1}>0$ (or $\left.<0\right) \quad \forall \varphi \in \operatorname{Ker}(-\Delta+V), \varphi \neq 0$.

Under these assumptions, by using bounded domain approximation techniques, Tehrani [37] proved the existence of at least one solution.

In this paper, we prove the existence of two solutions for problem (2.2), provided that both $a(x)$ and $V(x)$ change sign in $\mathbb{R}^{N}$. We consider two classes of assumptions on the indefinite non-symmetric potentials $a(x)$ and $V(x)$.

First class: We suppose that $V$ satisfies $\left(H_{1}\right)$ and the following hypotheses:

$\left(H_{3}\right) \quad V \in L^{\infty}\left(\mathbb{R}^{N}\right)$ and there exist $R_{0}, \beta>0$ such that

$$
V(x) \geq \beta, \quad \forall|x| \geq R_{0} .
$$

$\left(H_{4}\right)$ the linear problem

$$
\left\{\begin{array}{l}
-\Delta u+V(x) u=0, \\
u \in H^{1}\left(\mathbb{R}^{N}\right)
\end{array}\right.
$$

has not a nontrivial solution.

We suppose that $a$ satisfies:

$$
\left(A_{1}\right) \quad a \in L^{\infty}\left(\mathbb{R}^{N}\right)
$$

and there exist $\alpha, R_{1}>0$ such that

$$
a(x) \leq-\alpha, \quad \forall|x| \geq R_{1} .
$$

Second class: We assume that $a$ and $V$ satisfy $\left(H_{1}\right),\left(H_{3}\right),\left(N^{ \pm}\right)$and the following conditions:

$\left(\overline{A_{1}}\right) \quad a \in L^{\frac{2}{1-q}}\left(\mathbb{R}^{N}\right), \quad 0<q<1$.

$\left(H_{2}\right) \quad 0 \in \sigma_{d}(-\Delta+V)$,

where $\sigma_{d}$ is the discrete spectrum of $L=-\Delta+V(x)$.

We consider the functional $I: Y \rightarrow \mathbb{R}$, where $Y=H^{1}\left(\mathbb{R}^{N}\right) \cap L^{q+1}\left(\mathbb{R}^{N}\right)$ or $Y=$ $H^{1}\left(\mathbb{R}^{N}\right)$. The critical points of $I$ are weak solutions of problem (2.2).

The main results in this paper are the following.

Theorem 2.1 Assume that hypotheses $(F),\left(A_{1}\right),\left(H_{1}\right),\left(H_{3}\right)$ and $\left(H_{4}\right)$ hold. Then there exists $m_{0}>0$ such that if

$$
\|f\|_{2}^{2}+\left\|a^{+}+\chi_{B\left(0, R_{1}\right)}\right\|_{\frac{2}{2^{*}-q-1}}^{\frac{2}{1-q}}<m_{0},
$$

then problem (2.2) has two solutions $U_{1}, U_{2} \in E$ with $I\left(U_{1}\right)<0$ and $I\left(U_{2}\right)>0$. One of these solutions is nonnegative.

Theorem 2.2 Assume that $(F),\left(\overline{A_{1}}\right),\left(H_{1}\right),\left(H_{2}\right),\left(H_{3}\right)$ and $\left(N^{ \pm}\right)$hold. Then there exists $m_{1}>0$ such that if

$$
\|f\|_{2}^{2}+\left\|a^{+}+\chi_{B\left(0, R_{0}\right)}\right\|_{\frac{2}{1-q}}^{\frac{2}{1-q}}<m_{1},
$$


then problem (2.2) admits two solutions $V_{1}, V_{2} \in H^{1}\left(\mathbb{R}^{N}\right)$ with $I\left(V_{1}\right)<0$ and $I\left(V_{2}\right)>0$. One of these solutions is nonnegative.

Remark 2.3 In fact, the assumptions

$$
\|f\|_{2}^{2}+\left\|a^{+}+\chi_{B\left(0, R_{1}\right)}\right\|_{\frac{2}{2^{*}-q-1}}^{\frac{2}{1-q}}<m_{0}
$$

and

$$
\|f\|_{2}^{2}+\left\|a^{+}+\chi_{B\left(0, R_{0}\right)}\right\|_{\frac{2}{1-q}}^{\frac{2}{1-q}}<m_{1}
$$

are necessary only to guarantee that $I(u) \geq c>0$ on the sphere in $Y$ (see Lemmas 3.3 and 3.4). Thus these hypotheses can be removed if there are other ways to get $I(u) \geq c>0$ on the sphere in $Y$.

We divide our paper into four sections. In Sect. 2, we give some notations and preliminary results. In Sects. 3 and 4, we prove Theorems 2.1 and 2.2.

The main difficulties that arise in treating this class of nonhomogeneous Schrödinger equations (2.2) are the following: (i) the lack of compactness due to the unboundedness of the domain; (ii) the sign-changing of potentials $a(x)$ and $V(x)$. To avoid the first difficulty, we employ the Del Pino and Felmer method [23]. To overcome the second difficulty, we control the positive mass in relation to the negative mass of the potentials $a(x)$ and $V(x)$. The key tool for obtaining the multiplicity of solutions is a suitable recurrent variational method.

\section{Notations and preliminaries}

We will use the following notations:

$\|u\|_{m}=\left(\int_{\mathbb{R}^{N}}|u|^{m} d x\right)^{\frac{1}{m}}, \quad \forall 1 \leq m<\infty ;$

$B(0, R)$ denotes the ball centered in zero of radius $R>0$ and $B^{c}(0, R)=\mathbb{R}^{N} \backslash B(0, R)$;

$C_{S}$ is the best Sobolev constant:

$$
\forall u \in H^{1}\left(\mathbb{R}^{N}\right),\|u\|_{2^{*}} \leq C_{S}\|\nabla u\|_{2} ;
$$

$F^{\prime}$ : the Fréchet derivative of $F$ at $u$.

Let

$$
E=H^{1}\left(\mathbb{R}^{N}\right) \cap L^{q+1}\left(\mathbb{R}^{N}\right) .
$$

If we equip $E$ with the norm

$$
\|u\|=\|\nabla u\|_{2}+\|u\|_{q+1},
$$

then $E$ becomes a reflexive Banach space. On the Sobolev space $H^{1}\left(\mathbb{R}^{N}\right)$ we consider the usual norm

$$
\|u\|_{H}=\|u\|_{2}+\|\nabla u\|_{2} .
$$

Define the following energy functional on $Y\left(Y=E\right.$ or $\left.H^{1}\left(\mathbb{R}^{N}\right)\right)$ :

$$
\begin{aligned}
I(u)=\frac{1}{2} & \int_{\mathbb{R}^{N}}\left(|\nabla u|^{2}+V(x) u^{2}\right) d x-\frac{1}{q+1} \int_{\mathbb{R}^{N}} a(x)|u|^{q+1} d x \\
& -\int_{\mathbb{R}^{N}} f(x) u d x .
\end{aligned}
$$


Under suitable assumptions on $a, V$ and $f$ (to be fixed later), the functional $I$ is well defined, of class $C^{1}$ on $Y$ and any critical point of $I$ is a weak solution of problem (2.2).

We recall that a Palais-Smale sequence for the functional $I$, for short we write (PS)sequence, is a sequence $\left(u_{n}\right)_{n} \in Y$ such that

$$
\left(I\left(u_{n}\right)\right)_{n} \text { is bounded and }\left\|I^{\prime}\left(u_{n}\right)\right\|_{Y^{\prime}} \rightarrow 0 .
$$

The functional $I$ is said to satisfy the Palais-Smale condition if any (PS)-sequence has a convergent subsequence in $Y$.

In the sequel we need the following auxiliary results.

Lemma 3.1 Let $x$ and $y$ be arbitrary real numbers. Then there exists $c>0$ such that

$$
|| x+\left.y\right|^{q+1}-|x|^{q+1}-\left.|y|^{q+1}|\leq c| x\right|^{q} y,
$$

for $0<q<1$.

Proof If $x=0$, the inequality (3.2) is trivial.

Suppose that $x \neq 0$. Let $f: \mathbb{R} \backslash\{0\} \mapsto \mathbb{R}$ defined by

$$
f(t)=\frac{|1+t|^{q+1}-|t|^{q+1}-1}{|t|} .
$$

Then $\lim _{|t| \rightarrow+\infty} f(t)=0$ and $\lim _{t \rightarrow 0^{ \pm}} f(t)= \pm(q+1)$, so there exists constant $c>0$ such that $|f(t)| \leq c, \quad \forall t \in \mathbb{R} \backslash\{0\}$. In particular $\left|f\left(\frac{y}{x}\right)\right| \leq c$, so

$$
|| 1+\left.\frac{y}{x}\right|^{q+1}-\left|\frac{y}{x}\right|^{q+1}-1|\leq c| \frac{y}{x} \mid .
$$

Multiplying by $|x|^{q+1}$, we obtain the desired result.

Lemma 3.2 Assume that $\left(\overline{A_{1}}\right)$ holds. If $u_{n} \rightarrow u$ weakly in $H^{1}\left(\mathbb{R}^{N}\right)$, then there exists a subsequence of $\left(u_{n}\right) \in H^{1}\left(\mathbb{R}^{N}\right)$, also denoted $\left(u_{n}\right)$, such that

$$
\lim _{n \rightarrow+\infty} \int_{\mathbb{R}^{N}} a(x)\left|u_{n}-u\right|^{q+1} d x=0 .
$$

Proof Since $a \in L^{\frac{2}{1-q}}\left(\mathbb{R}^{N}\right)$, then for every $\epsilon>0$ there exists $R_{2}>0$ such that

$$
\left(\int_{|x|>R_{2}}|a(x)|^{\frac{2}{1-q}} d x\right)^{\frac{1-q}{2}}<\epsilon .
$$

Since $u_{n} \rightarrow u$ weakly in $H^{1}\left(\mathbb{R}^{N}\right), u_{n} \rightarrow u$ strongly in $L_{\text {loc }}^{s}\left(\mathbb{R}^{N}\right), 2 \leq s<2^{*}$, then

$$
\left(\int_{|x|<R_{2}}\left|u_{n}-u\right|^{2} d x\right)^{\frac{q+1}{2}}<\epsilon .
$$

Observe that by Hölder's inequality we have

$$
\begin{aligned}
\int_{\mathbb{R}^{N}} a(x)\left|u_{n}-u\right|^{q+1} d x= & \int_{|x| \leq R_{2}} a(x)\left|u_{n}-u\right|^{q+1} d x \\
& +\int_{|x|>R_{2}} a(x)\left|u_{n}-u\right|^{q+1} d x<c \epsilon,
\end{aligned}
$$

hence $\lim _{n \rightarrow+\infty} \int_{\mathbb{R}^{N}} a(x)\left|u_{n}-u\right|^{q+1} d x=0$. 
Lemma 3.3 Assume that hypotheses $\left(A_{1}\right),\left(H_{3}\right)$ and $(F)$ hold. Then there exist $\rho_{0}, \alpha_{0}$, $m_{0}>0$ such that $I(u) \geq \alpha_{0}>0$ for all $u \in E,\|u\|=\rho_{0}$ and $\|f\|_{2}^{2}+$ $\left\|a^{+}+\chi_{B\left(0, R_{1}\right)}\right\|_{\frac{2}{1-q}}^{\frac{2}{2^{*}-q-1}}<m_{0}$.

Proof Let $u \in E$. By Hölder and Sobolev inequalities, we have

$$
\begin{aligned}
\|u\|_{2}^{2} & \leq\|u\|_{q+1}^{2 r}\|u\|_{2^{*}}^{2(1-r)} \leq C_{s}^{2(1-r)}\|u\|_{q+1}^{2 r}\|\nabla u\|_{2}^{2(1-r)} \\
& \leq \frac{\min (\alpha, 1)}{(q+1)\left(\|V\|_{\infty}+1\right)}\|u\|_{q+1}^{q+1}+c\|\nabla u\|_{2}^{p}
\end{aligned}
$$

with

$$
\left.r=\frac{\left(2^{*}-2\right)(q+1)}{2\left(2^{*}-q-1\right)} \in\right] 0,1\left[\text { and } p=\frac{2(1-r)(q+1)}{q+1-2 r}>2 .\right.
$$

Then from $(F),\left(H_{3}\right)$ and $\left(A_{1}\right)$ we infer that

$$
\begin{aligned}
& I(u)=\frac{1}{2} \int_{\mathbb{R}^{N}}\left(|\nabla u|^{2}+V(x) u^{2}\right) d x-\frac{1}{q+1} \int_{\mathbb{R}^{N}}\left(a^{+}+\chi_{B\left(0, R_{1}\right)}\right)(x)|u|^{q+1} d x \\
& +\frac{1}{q+1} \int_{\mathbb{R}^{N}}\left(a^{-}+\chi_{B\left(0, R_{1}\right)}\right)(x)|u|^{q+1} d x-\int_{\mathbb{R}^{N}} f(x) u d x \\
& \geq \frac{1}{2}\left(\|\nabla u\|_{2}^{2}-\left(\|V\|_{\infty}+1\right)\|u\|_{2}^{2}\right)+\frac{\min (\alpha, 1)}{q+1}\|u\|_{q+1}^{q+1} \\
& -\frac{1}{q+1}\left\|a^{+}+\chi_{B\left(0, R_{1}\right)}\right\|_{\frac{2^{*}}{2^{*}-q-1}}\|u\|_{2^{*}}^{q+1}-\frac{1}{2}\|f\|_{2}^{2} \\
& \geq \frac{1}{2}\|\nabla u\|_{2}^{2}+\frac{\min (\alpha, 1)}{2(q+1)}\|u\|_{q+1}^{q+1}-c\|\nabla u\|_{2}^{p}-\frac{C_{s}^{q+1}}{q+1}\left\|a^{+}+\chi_{B\left(0, R_{1}\right)}\right\|_{\frac{2^{*}}{2^{*}-q-1}}\|\nabla u\|_{2}^{q+1} \\
& -\frac{1}{2}\|f\|_{2}^{2} \\
& \geq \frac{1}{4}\|\nabla u\|_{2}^{2}+\frac{\min (\alpha, 1)}{2(q+1)}\|u\|_{q+1}^{q+1}-c\|\nabla u\|_{2}^{p} \\
& -(2(q+1))^{\frac{q+1}{1-q}}\left(\frac{C_{s}^{q+1}}{q+1}\right)^{\frac{2}{1-q}}\left(\frac{1-q}{2}\right)\left\|a^{+}+\chi_{B\left(0, R_{1}\right)}\right\|_{\frac{2^{*}}{2^{*}-q-1}}^{\frac{2}{1-q}}-\frac{1}{2}\|f\|_{2}^{2} .
\end{aligned}
$$

Since $p>2$, for $\|\nabla u\|_{2} \leq s$ (small enough) we have

$$
\frac{1}{4}\|\nabla u\|_{2}^{2}-c\|\nabla u\|_{2}^{p} \geq \frac{1}{8}\|\nabla u\|_{2}^{2} .
$$

Next, using the inequality

$$
\frac{1}{2}(x+y)^{2} \leq x^{2}+y^{q+1} \quad \forall x \geq 0, \quad 0 \leq y \leq 1,
$$

we obtain that for $\|\nabla u\|_{2} \leq s$ and $\|u\|_{q+1} \leq 1$, we have

$$
\begin{aligned}
I(u) \geq & \frac{\|\nabla u\|_{2}^{2}}{8}+\frac{\min (\alpha, 1)}{2(q+1)}\|u\|_{q+1}^{q+1}-\frac{1}{2}\|f\|_{2}^{2} \\
& -(2(q+1))^{\frac{q+1}{1-q}}\left(\frac{C_{s}^{q+1}}{q+1}\right)^{\frac{2}{1-q}}\left(\frac{1-q}{2}\right)\left\|a^{+}+\chi_{B\left(0, R_{1}\right)}\right\|_{\frac{2^{*}}{2^{*}-q-1}}^{\frac{2}{1-q}} \\
\geq & c_{0}\|u\|^{2}-\frac{1}{2}\|f\|_{2}^{2} \\
& -(2(q+1))^{\frac{q+1}{1-q}}\left(\frac{C_{s}^{q+1}}{q+1}\right)^{\frac{2}{1-q}}\left(\frac{1-q}{2}\right)\left\|a^{+}+\chi_{B\left(0, R_{1}\right)}\right\|_{\frac{2}{2^{*}-q-1}}^{\frac{2}{1-q}},
\end{aligned}
$$


where $c_{0}=\min \left(\frac{1}{16}, \frac{\min (\alpha, 1)}{4(q+1)}\right)$.

Set $\rho_{0}=\min (s, 1)$. Then by $(3.4)$ and for

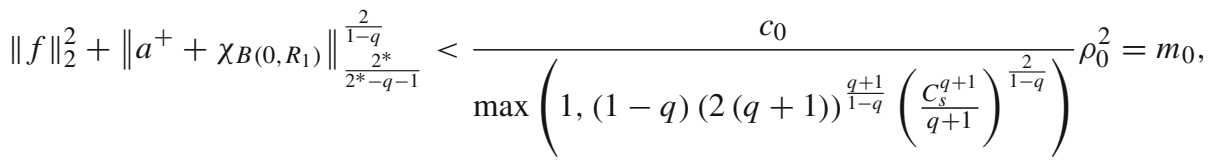

we infer that

$$
I(u) \geq c_{0} \frac{\rho_{0}^{2}}{2}=\alpha_{0}, \text { for }\|u\|=\rho_{0} .
$$

The proof is completed.

Lemma 3.4 Suppose that hypotheses $(F),\left(\overline{A_{1}}\right)$ and $\left(H_{3}\right)$ are satisfied. Then there exist $\rho_{1}, \quad \alpha_{1}, \quad m_{1}>0$ such that $I(u) \geq \alpha_{1}>0$ for all $u \in H^{1}\left(\mathbb{R}^{N}\right),\|u\|_{H}=\rho_{1}$ and

$$
\|f\|_{\frac{2 N}{N+2}}^{2}+\left\|a^{+}+\chi_{B\left(0, R_{0}\right)}\right\|_{\frac{2}{1-q}}^{\frac{2}{1-q}}<m_{1} .
$$

Proof Combining hypothesis $\left(H_{3}\right)$ with Hölder and Sobolev inequalities, we obtain

$$
\begin{aligned}
& \int_{\mathbb{R}^{N}} V^{-}(x) u^{2} d x \leq\|V\|_{\infty} \int_{\mathbb{R}^{N}}\left(\left(a^{-}+\chi_{B\left(0, R_{0}\right)}\right)(x)\right)^{\frac{2 r}{q+1}} u^{2} d x \\
& \quad \leq\|V\|_{\infty}\left(\int_{\mathbb{R}^{N}}\left(a^{-}+\chi_{B\left(0, R_{0}\right)}\right)(x)|u|^{q+1} d x\right)^{\frac{2 r}{q+1}}\|u\|_{2^{*}}^{2(1-r)} \\
& \quad \leq\|V\|_{\infty} C_{s}^{2(1-r)}\left(\int_{\mathbb{R}^{N}}\left(a^{-}+\chi_{B\left(0, R_{0}\right)}\right)(x)|u|^{q+1} d x\right)^{\frac{2 r}{q+1}}\|\nabla u\|_{2}^{2(1-r)} \\
& \quad \leq \frac{1}{(q+1)} \int_{\mathbb{R}^{N}}\left(a^{-}+\chi_{B\left(0, R_{0}\right)}\right)(x)|u|^{q+1} d x+c\|\nabla u\|_{2}^{p}
\end{aligned}
$$

and

$$
\begin{aligned}
\int_{\mathbb{R}^{N}} & \left(|\nabla u|^{2}+u^{2}\right) d x=\int_{\mathbb{R}^{N}}|\nabla u|^{2}+\int_{B\left(0, R_{0}\right)} u^{2} d x+\int_{B^{c}\left(0, R_{0}\right)} u^{2} d x \\
\leq & \int_{\mathbb{R}^{N}}|\nabla u|^{2} d x+C_{s}^{2}\left(\operatorname{meas}\left(B\left(0, R_{0}\right)\right)\right)^{\frac{2}{N}} \int_{\mathbb{R}^{N}}|\nabla u|^{2} d x \\
\quad & +\frac{1}{\beta} \int_{B^{c}\left(0, R_{0}\right)} V^{+}(x) u^{2} d x \\
\leq & \max \left\{\max \left(1, C_{s}^{2}\left(\operatorname{meas}\left(B\left(0, R_{0}\right)\right)\right)^{\frac{2}{N}}\right), \frac{1}{\beta}\right\} \\
\quad & \times\left(\int_{\mathbb{R}^{N}}|\nabla u|^{2}+\int_{B^{c}\left(0, R_{0}\right)} V^{+}(x) u^{2} d x\right) .
\end{aligned}
$$


Using (3.5), we have

$$
\begin{aligned}
I(u)= & \frac{1}{2} \int_{\mathbb{R}^{N}}\left(|\nabla u|^{2}+V^{+}(x) u^{2}\right) d x-\frac{1}{2} \int_{\mathbb{R}^{N}} V^{-}(x) u^{2} d x \\
& +\frac{1}{q+1} \int_{\mathbb{R}^{N}}\left(a^{-}+\chi_{B\left(0, R_{0}\right)}\right)(x)|u|^{q+1} d x \\
& -\frac{1}{q+1} \int_{\mathbb{R}^{N}}\left(a^{+}+\chi_{B\left(0, R_{0}\right)}\right)(x)|u|^{q+1} d x-\int_{\mathbb{R}^{N}} f(x) u d x \\
\geq & \frac{1}{4}\|\nabla u\|_{2}^{2}-c\|\nabla u\|_{2}^{p}+\frac{1}{2} \int_{B_{\left(0, R_{0}\right)}^{c}} V^{+}(x) u^{2} d x \\
& +\frac{1}{2(q+1)} \int_{\mathbb{R}^{N}}\left(a^{-}+\chi_{B\left(0, R_{0}\right)}\right)(x)|u|^{q+1} d x \\
& -\left\|a^{+}+\chi_{B\left(0, R_{0}\right)}\right\|_{\frac{2}{1-q}}\|u\|_{H}^{q+1}-C_{s}^{2}\|f\|_{\frac{2 N}{N+2}}^{2} \\
\geq & \frac{1}{4}\|\nabla u\|_{2}^{2}-c\|\nabla u\|_{2}^{p}+\frac{1}{2} \int_{B^{c}\left(0, R_{0}\right)} V^{+}(x) u^{2} d x \\
& -\left\|a^{+}+\chi_{B\left(0, R_{0}\right)}\right\|_{\frac{2}{1-q}}\|u\|_{H}^{q+1}-C_{s}^{2}\|f\|_{\frac{2 N}{N+2}}^{2} .
\end{aligned}
$$

Using (3.6) and for $\|\nabla u\|_{2} \leq \rho_{1}$ ( $\rho_{1}$ small enough), we infer that

$$
\begin{aligned}
I(u) \geq & \frac{1}{8}\left(\int_{\mathbb{R}^{N}} \nabla u^{2} d x+\int_{B^{c}\left(0, R_{0}\right)} V^{+}(x) u^{2} d x\right) \\
& -\left\|a^{+}+\chi_{B\left(0, R_{0}\right)}\right\|_{\frac{2}{1-q}}\|u\|_{H}^{q+1}-C_{s}^{2}\|f\|_{\frac{2 N}{N+2}}^{2} \\
\geq & c_{1}\|u\|_{H}^{2}-\frac{1-q}{2}\left(\frac{2}{c_{1}}\right)^{\frac{q+1}{1-q}}\left(\frac{q+1}{2}\right)^{\frac{1+q}{1-q}}\left\|a^{+}+\chi_{B\left(0, R_{0}\right)}\right\|_{\frac{2}{1-q}}^{\frac{2}{1-q}}-C_{s}^{2}\|f\|_{\frac{2 N}{N+2}}^{2},
\end{aligned}
$$

where

$$
c_{1}=\frac{1}{8 \max \left(\max \left(1, C_{s}^{2}\left(\operatorname{meas}\left(B\left(0, R_{0}\right)\right)\right)^{\frac{2}{N}}\right), \frac{1}{\beta}\right)} .
$$

Set

$$
m_{1}=\frac{c_{1}}{2 \max \left(2 C_{s}^{2},(1-q)\left(\frac{2}{c_{1}}\right)^{\frac{1+q}{1-q}}\left(\frac{q+1}{2}\right)^{\frac{1+q}{1-q}}\right)} .
$$

If $\|f\|_{\frac{2 N}{N+2}}^{2}+\left\|a^{+}+\chi_{B\left(0, R_{0}\right)}\right\|_{\frac{2}{1-q}}^{\frac{2}{1-q}}<m_{1}$, we have

$$
I(u) \geq \frac{c_{1}}{2} \rho_{1}^{2}=\alpha_{1}, \text { for }\|u\|_{H}=\rho_{1} .
$$

This completes the proof.

\section{Proof of Theorem 2.1}

We split the proof into several steps. We first establish the existence of first solution with negative energy. Next, we show that problem (2.2) has a weak solution with positive energy. 


\subsection{Existence of a nonnegative solution}

Consider the minimization problem

$$
M_{1}=\inf _{u \in B\left(0, \rho_{0}\right)} I(u),
$$

where $\rho_{0}$ is defined in Lemma 3.3.

Lemma 4.1 Assume that hypotheses $\left(A_{1}\right),\left(H_{3}\right)$ and $(F)$ hold. Then $-\infty<M_{1}<0$.

Proof By Lemma 3.3 we have $M_{1}>-\infty$.

Using hypothesis $(F)$, there is a function $\psi \in E$ such that $\int_{\mathbb{R}^{N}} f(x) \psi d x>0$. For $t>0$, we have

$$
\begin{aligned}
I(t \psi)= & \frac{t^{2}}{2} \int_{\mathbb{R}^{N}}\left(|\nabla \psi|^{2}+V(x) \psi^{2}\right) d x \\
& -\frac{t^{q+1}}{q+1} \int_{\mathbb{R}^{N}} a(x)|\psi|^{q+1} d x-t \int_{\mathbb{R}^{N}} f(x) \psi d x \\
< & 0 \text { for } t>0 \text { small enough. }
\end{aligned}
$$

Hence $M_{1}<0$. Thus we conclude the proof.

Theorem 4.2 Assume that hypotheses $(F),\left(A_{1}\right)$ and $\left(H_{3}\right)$ hold. Then there exists a weak nonnegative solution $U_{1} \in E$ of problem (2.2), $I\left(U_{1}\right)=M_{1}$.

Proof Let $\left(u_{n}\right)_{n}$ be a minimizing sequence of problem (4.1). Since $\left(u_{n}\right) \in B\left(0, \rho_{0}\right)$, we can extract a subsequence, also denoted by $\left(u_{n}\right)$, such that $u_{n} \rightarrow U_{1}$ in $E, u_{n} \rightarrow U_{1}$ in $L_{l o c}^{m}\left(\mathbb{R}^{N}\right)$ for all $1 \leq m<2^{*}$ and $u_{n} \rightarrow U_{1}$ a.e. in $\mathbb{R}^{N}$.

Setting $v_{n}=u_{n}-U_{1} \rightarrow 0$ in $E$, we have

$$
\begin{aligned}
I\left(u_{n}\right)= & \frac{1}{2} \int_{\mathbb{R}^{N}}\left(\left|\nabla v_{n}\right|^{2}+V(x) v_{n}^{2}\right) d x+\frac{1}{2} \int_{\mathbb{R}^{N}}\left(\left|\nabla U_{1}\right|^{2}+V(x) U_{1}^{2}\right) d x \\
& +\int_{\mathbb{R}^{N}}\left(\nabla v_{n} \nabla U_{1}+V(x) v_{n} U_{1}\right) d x \\
& -\frac{1}{q+1} \int_{\mathbb{R}^{N}} a(x)\left(\left|u_{n}\right|^{q+1}-\left|U_{1}\right|^{q+1}\right) d x-\frac{1}{q+1} \int_{\mathbb{R}^{N}} a(x)\left|U_{1}\right|^{q+1} d x \\
& -\int_{\mathbb{R}^{N}} f(x) v_{n} d x-\int_{\mathbb{R}^{N}} f(x) U_{1} d x .
\end{aligned}
$$

Since $v_{n} \rightarrow 0$ in $E$, we obtain

$$
\int_{\mathbb{R}^{N}}\left(\nabla v_{n} \nabla U_{1}+V(x) v_{n} U_{1}\right) d x \rightarrow 0 \text { and } \int_{\mathbb{R}^{N}} f(x) v_{n} d x \rightarrow 0 .
$$

By Lemma 3.1 we have

$$
\left.\int_{\mathbb{R}^{N}}|a(x)||| u_{n}\right|^{q+1}-\left.\left(\left|U_{1}\right|^{q+1}+\left|v_{n}\right|^{q+1}\right)\left|d x \leq c \int_{\mathbb{R}^{N}}\right| a(x)|| U_{1}\right|^{q}\left|v_{n}\right| \rightarrow 0 .
$$

From $\left(H_{3}\right)$ and $\left(A_{1}\right)$, we deduce that

$$
\int_{\mathbb{R}^{N}} V^{-}(x) v_{n}^{2} d x \rightarrow 0 \text { and } \int_{\mathbb{R}^{N}}\left(a^{+}(x)+\chi_{B\left(0, R_{1}\right)}\right)\left|v_{n}\right|^{q+1} d x \rightarrow 0 .
$$

Using relations (4.4), (4.5), (4.6) and passing to the limit in (4.3), we get

$$
\begin{aligned}
M_{1} & =I\left(U_{1}\right)+\lim _{n \rightarrow+\infty}\left(\frac{1}{2} \int_{\mathbb{R}^{N}}\left|\nabla v_{n}\right|^{2}+V^{+}(x) v_{n}^{2}+\frac{1}{q+1}\left(a^{-}(x)+\chi_{B\left(0, R_{1}\right)}\right)\left|v_{n}\right|^{q+1}\right) d x \\
& \geq I\left(U_{1}\right)+\lim _{n \rightarrow+\infty}\left(\frac{1}{2}\left\|\nabla v_{n}\right\|_{2}^{2}+\frac{\min (\alpha, 1)}{q+1}\left\|v_{n}\right\|_{q+1}^{q+1}\right) \geq M_{1} .
\end{aligned}
$$


It follows that $v_{n} \rightarrow 0$ in $E, M_{1}=I\left(U_{1}\right)<0, U_{1} \in B\left(0, \rho_{0}\right), I^{\prime}\left(U_{1}\right)=0$. Thus $U_{1}$ is a weak solution of problem (2.2). Since $M_{1} \leq I\left(\left|u_{n}\right|\right) \leq I\left(u_{n}\right)$, then $\left(\left|u_{n}\right|\right)$ is also a minimizing sequence of problem (4.1). We deduce that we can suppose that $U_{1} \geq 0$ a.e. in $\mathbb{R}^{N}$.

\subsection{Existence of a second solution with positive energy solution}

We start this subsection by showing that the functional $I$ satisfies the Palais-Smale condition. For this purpose, we need the following auxiliary properties.

Lemma 4.3 Assume that hypotheses $\left(A_{1}\right),\left(H_{3}\right),\left(H_{4}\right)$ and $(F)$ hold. Then any $(P S)$-sequence of $I$ is bounded in $E$.

Proof Let $\left(u_{n}\right) \in E$ be a (PS)-sequence of $I$. We argue by contradiction, assuming that $\left\|u_{n}\right\|=t_{n} \rightarrow+\infty$. Re-normalizing, we set $v_{n}=\frac{u_{n}}{t_{n}}$. Thus up to a subsequence, $v_{n} \rightarrow v$ in E.

We claim that $v=0$. For this purpose, we take $\varphi \in C_{c}^{\infty}\left(\mathbb{R}^{N}\right)$. Since $\left(u_{n}\right)$ is a $(P S)$ sequence of $I$, we have

$$
\begin{aligned}
\left\langle I^{\prime}\left(u_{n}\right), \varphi\right\rangle= & \int_{\mathbb{R}^{N}}\left(\nabla u_{n} \nabla \varphi+V(x) u_{n} \varphi\right) d x-\int_{\mathbb{R}^{N}} a(x)\left|u_{n}\right|^{q-1} u_{n} \varphi d x \\
& -\int_{\mathbb{R}^{N}} f(x) \varphi d x=o(1)\|\varphi\| .
\end{aligned}
$$

Dividing relation (4.7) by $t_{n}$, we obtain

$$
\int_{\mathbb{R}^{N}}\left(\nabla v_{n} \nabla \varphi+V(x) v_{n} \varphi\right) d x-\int_{\mathbb{R}^{N}} a(x) \frac{\left|u_{n}\right|^{q-1} u_{n}}{t_{n}} \varphi d x-\int_{\mathbb{R}^{N}} \frac{f(x) \varphi}{t_{n}} d x=o(1) .
$$

Using $\left(A_{1}\right)$ and Hölder's inequality, we deduce that

$$
\int_{\mathbb{R}^{N}} a(x) \frac{\left|u_{n}\right|^{q-1} u_{n}}{t_{n}} \varphi d x \leq\|a\|_{\infty} \frac{\left\|u_{n}\right\|_{2^{*}}^{q}}{t_{n}}\|\varphi\|_{\frac{2^{*}}{2^{*}-q}} \leq c\|a\|_{\infty} \frac{\left\|u_{n}\right\|^{q}}{t_{n}}\|\varphi\|_{\frac{2^{*}}{2^{*}-q}} .
$$

Thus since $0<q<1$, we have

$$
\int_{\mathbb{R}^{N}} a(x) \frac{\left|u_{n}\right|^{q-1} u_{n}}{t_{n}} \varphi d x \rightarrow 0 .
$$

As a consequence of hypothesis $(F)$, we have

$$
\int_{\mathbb{R}^{N}} \frac{f(x) \varphi}{t_{n}} d x \rightarrow 0 \text { as } n \rightarrow+\infty .
$$

From (4.9), (4.10) and passing to the limit in relation (4.8), we infer that

$$
\int_{\mathbb{R}^{N}}(\nabla v \nabla \varphi+V(x) v \varphi) d x=0 .
$$

Using now $\left(H_{4}\right)$, we conclude that $v=0$ a.e. in $\mathbb{R}^{N}$. This proves our claim.

Substituting $\varphi=v_{n}$ in relation (4.8), we obtain

$$
\begin{gathered}
\int_{\mathbb{R}^{N}}\left|\nabla v_{n}\right|^{2} d x+\int_{\mathbb{R}^{N}}\left(V^{+}+\chi_{B\left(0, R_{0}\right)}\right)(x) v_{n}^{2} d x-\int_{\mathbb{R}^{N}}\left(V^{-}(x)+\chi_{B\left(0, R_{0}\right)}(x)\right) v_{n}^{2} d x \\
-\int_{\mathbb{R}^{N}} \frac{a^{+}(x)\left|u_{n}\right|^{q+1}}{t_{n}^{2}} d x+\int_{\mathbb{R}^{N}} \frac{a^{-}(x)\left|u_{n}\right|^{q+1}}{t_{n}^{2}} d x-\int_{\mathbb{R}^{N}} \frac{f(x) v_{n}}{t_{n}}=o(1) .
\end{gathered}
$$


It follows that

$$
\begin{array}{r}
\left\|\nabla v_{n}\right\|_{2}^{2}-\int_{\mathbb{R}^{N}}\left(V^{-}(x)+\chi_{B\left(0, R_{0}\right)}(x)\right) v_{n}^{2} d x \\
\quad-\int_{\mathbb{R}^{N}} \frac{a^{+}(x)\left|u_{n}\right|^{q+1}}{t_{n}^{2}} d x-\int_{\mathbb{R}^{N}} \frac{f(x) v_{n}}{t_{n}} \leq o(1) .
\end{array}
$$

Using now $\left(A_{1}\right),\left(H_{3}\right)$ and $(F)$, we obtain

$$
\begin{aligned}
& \int_{\mathbb{R}^{N}} \frac{a^{+}(x)\left|u_{n}\right|^{q+1}}{t_{n}^{2}} d x \rightarrow 0 \\
& \int_{\mathbb{R}^{N}}\left(V^{-}+\chi_{B\left(0, R_{0}\right)}\right)(x) v_{n}^{2} d x \rightarrow 0 \\
& \int_{\mathbb{R}^{N}} \frac{f(x) v_{n}}{t_{n}} \rightarrow 0 .
\end{aligned}
$$

Combining (4.11) and (4.12), we conclude that

$$
\lim _{n \rightarrow+\infty}\left\|\nabla v_{n}\right\|_{2}=0 .
$$

On the other hand, since $\left(u_{n}\right)$ is a (PS)-sequence of $I$, we have

$$
\frac{\int_{\mathbb{R}^{N}}\left(\left|\nabla u_{n}\right|^{2}+V(x) u_{n}^{2}\right) d x}{t_{n}^{q+1}}=\frac{\int_{\mathbb{R}^{N}} a(x)\left|u_{n}\right|^{q+1} d x}{t_{n}^{q+1}}+\frac{\int_{\mathbb{R}^{N}} f(x) u_{n}}{t_{n}^{q+1}}+o(1) .
$$

Therefore

$$
\begin{aligned}
o(1)=\frac{I\left(u_{n}\right)}{t_{n}^{q+1}}= & \left(\frac{1}{2}-\frac{1}{q+1}\right) \int_{\mathbb{R}^{N}} a(x)\left|v_{n}\right|^{q+1} d x-\frac{1}{2 t_{n}^{q}} \int_{\mathbb{R}^{N}} f(x) v_{n} \\
= & \left(\frac{1}{q+1}-\frac{1}{2}\right) \int_{\mathbb{R}^{N}}\left(a^{-}+\chi_{B\left(0, R_{1}\right)}\right)(x)\left|v_{n}\right|^{q+1} d x \\
& -\left(\frac{1}{q+1}-\frac{1}{2}\right) \int_{\mathbb{R}^{N}}\left(a^{+}+\chi_{B\left(0, R_{1}\right)}\right)(x)\left|v_{n}\right|^{q+1} d x \\
& -\frac{1}{2 t_{n}^{q}} \int_{\mathbb{R}^{N}} f(x) v_{n} .
\end{aligned}
$$

From $\left(A_{1}\right),(F)$ and (4.14), we infer that

$$
\lim _{n \rightarrow+\infty}\left\|v_{n}\right\|_{q+1}=0 .
$$

Combining (4.13) and (4.15), we conclude that $v_{n} \rightarrow 0$ in $E$ as $n \rightarrow+\infty$, which contradicts $\left\|v_{n}\right\|=1$. The proof is completed.

Lemma 4.4 Assume that hypotheses $\left(A_{1}\right),\left(H_{1}\right),\left(H_{3}\right),\left(H_{4}\right)$ and $(F)$ hold. Then I satisfies the $(P S)$ condition.

Proof Let $\left(u_{n}\right) \in E$ be a $(P S)$ sequence such that

$$
I\left(u_{n}\right) \rightarrow M_{2} \text { and } I^{\prime}\left(u_{n}\right) \rightarrow 0 \text { in } E^{\prime} .
$$

Using Lemma 4.3, $\left(u_{n}\right)$ is bounded in $E$. Then up to a subsequence, $u_{n} \rightarrow U_{2}$ in $E, u_{n} \rightarrow U_{2}$ in $L_{\text {loc }}^{m}\left(\mathbb{R}^{N}\right)$ for all $1 \leq m<2^{*}$ and $u_{n} \rightarrow U_{2}$ a.e. in $\mathbb{R}^{N}$. According to [23], it is sufficient to prove that for any $\epsilon>0$, there exist $R_{2}>0$ and $n_{0} \in \mathbb{N}^{*}$ such that

$$
\int_{|x| \geq R_{2}}\left(\left|\nabla u_{n}\right|^{2}+\left|u_{n}\right|^{q+1}\right) d x \leq \epsilon, \quad \text { for all } R \geq R_{2} \text { and } n \geq n_{0} .
$$

Let $R \geq 1$ and $\phi_{R}$ be a smooth cut-off function such that $\phi_{R}=0$ on $B\left(0, \frac{R}{2}\right), \phi_{R}=1$ on $B^{c}(0, R), 0 \leq \phi_{R} \leq 1$ and there exists $c>0$ such that

$$
\left|\nabla \phi_{R}\right| \leq \frac{c}{R} \text {, for all } x \in \mathbb{R}^{N} \text {. }
$$


We remark that for any $u \in E$,

$$
\left\|\phi_{R} u\right\| \leq c\|u\| .
$$

Since $I^{\prime}\left(u_{n}\right) \rightarrow 0$ in $E^{\prime}$ then for any $\epsilon>0$ there exists $n_{0}>0$ such that

$$
\left|\left\langle I^{\prime}\left(u_{n}\right), \phi_{R} u_{n}\right\rangle\right| \leq c\left\|I^{\prime}\left(u_{n}\right)\right\|_{E^{\prime}}\left\|u_{n}\right\| \leq \frac{\epsilon}{3}, \quad \forall n \geq n_{0} .
$$

Thus for $n \geq n_{0}$,

$$
\begin{aligned}
& \int_{\mathbb{R}^{N}}\left(\left|\nabla u_{n}\right|^{2}+\left(a^{-}+\chi_{B\left(0, R_{1}\right)}\right)(x)\left|u_{n}\right|^{q+1}\right) \phi_{R} d x \\
& \leq \int_{\mathbb{R}^{N}} f(x) u_{n} \phi_{R} d x+\int_{\mathbb{R}^{N}} V^{-}(x) u_{n}^{2} \phi_{R} d x \\
& \quad-\int_{\mathbb{R}^{N}} u_{n} \nabla u_{n} \nabla \phi_{R} d x \\
& \quad+\int_{\mathbb{R}^{N}}\left(a^{+}(x)+\chi_{B\left(0, R_{1}\right)}\right)\left|u_{n}\right|^{q+1} \phi_{R} d x+\frac{\epsilon}{3} .
\end{aligned}
$$

By the Hölder inequality and (4.16), there exists $R_{3}>0$ such that

$$
\int_{\mathbb{R}^{N}} u_{n} \nabla u_{n} \nabla \phi_{R} d x \leq \frac{c}{R}<\frac{\epsilon}{3}, \text { for all } R \geq R_{3} .
$$

From $(F),\left(H_{3}\right)$ and $\left(A_{1}\right)$, there exists $R_{4}>0$ such that

$$
\begin{aligned}
& \int_{\mathbb{R}^{N}} f(x) u_{n} \phi_{R} d x+\int_{\mathbb{R}^{N}} V^{-}(x) u_{n}^{2} \phi_{R} d x+\int_{\mathbb{R}^{N}}\left(a^{+}+\chi_{B\left(0, R_{1}\right)}\right)(x)\left|u_{n}\right|^{q+1} d x \\
& \quad \leq c\left(\left\|f \phi_{R}\right\|_{2}+\left\|V^{-} \phi_{R}\right\|_{\frac{N}{2}}+\left\|\left(a^{+}+\chi_{B(0, R)}\right) \phi_{R}\right\|_{\frac{2^{*}}{2^{*}-q-1}}\right) \\
& \quad \leq \frac{\epsilon}{3} \text { for } R \geq R_{4} .
\end{aligned}
$$

Set $R_{2}=\max \left(R_{3}, R_{4}\right)$. Then by (4.18), (4.19) and (4.20) we deduce that

$$
\min (1, \min (\alpha, 1)) \int_{\mathbb{R}^{N}}\left(\left|\nabla u_{n}\right|^{2}+\left|u_{n}\right|^{q+1}\right) \phi_{R} d x \leq \epsilon, \quad \forall n \geq n_{0} \text { and } \forall R \geq R_{2} .
$$

This ends the proof.

By (4.2) and $\left(H_{1}\right)$, there exists $\varphi_{0}=t \phi \in B^{c}\left(0, \rho_{0}\right)$, with $t$ large enough, such that $I\left(\varphi_{0}\right)<0\left(\rho_{0}\right.$ is fixed by Lemma 3.3).

Consider the problem

$$
M_{0}=\inf _{\gamma \in \Gamma} \max _{t \in[0,1]} I(\gamma(t))
$$

where

$$
\Gamma=\left\{\gamma \in C([0,1], E), \gamma(0)=0 \text { and } \gamma(1)=\varphi_{0}\right\} .
$$

We observe that $M_{0}>0$. Indeed for $\gamma \in \Gamma$, we have $\gamma(0)=0$ and $\gamma(1) \notin B\left(0, \rho_{0}\right)$. Then there exists $r_{0} \in[0,1]$ such that $\left\|\gamma\left(r_{0}\right)\right\|=\rho_{0}$. By Lemma 3.3 we have $I\left(\gamma\left(r_{0}\right)\right)>\alpha_{0}$ and so $M_{0}>0$. Then, by Mountain-Pass theorem [5] and Lemma 4.4, we prove the existence of $U_{0} \in E$ such that $I\left(U_{0}\right)=M_{0}>0 . U_{0}$ is a weak solution of problem (2.2). 


\section{Proof of Theorem 2.2}

We first observe that $\left(H_{2}\right)$ and $\left(H_{3}\right)$ imply that the Schrödinger operator $L=-\Delta+V(x)$ is defined on $H^{2}\left(\mathbb{R}^{N}\right)$ and 0 is an isolated eigenvalue of finite multiplicity.

Let $H^{-}, H^{0}$ and $H^{+}$denote the negative, null and positive spaces of the quadratic form associated to the operator $L$. More precisely,

$$
H^{-}=\oplus_{\lambda_{i}<0} \operatorname{Ker}\left(L-\lambda_{i} I\right), \quad H^{0}=\operatorname{Ker}(L), \quad H^{+}=\oplus_{\lambda_{i}>0} \operatorname{Ker}\left(L-\lambda_{i} I\right) .
$$

We have the orthogonal decomposition $H^{1}\left(\mathbb{R}^{N}\right)=H^{-} \oplus H^{0} \oplus H^{+}$.

For $u \in E$, we denote by $u^{\mp}, u^{0}$ the orthogonal projections of $u$ on $H^{\mp}, H^{0}$ respectively. Then $u=u^{-}+u^{0}+u^{+}$. Moreover, there is an equivalent norm $\|\cdot\|_{X}$ on $H^{1}\left(\mathbb{R}^{N}\right)$ such that

$$
I(u)=\frac{1}{2}\left(\left\|u^{+}\right\|_{X}^{2}-\left\|u^{-}\right\|_{X}^{2}\right)-\frac{1}{q+1} \int_{\mathbb{R}^{N}} a(x)|u|^{q+1} d x-\int_{\mathbb{R}^{N}} f(x) u d x .
$$

For more details, we refer the reader to Costa and Tehrani [22].

\subsection{Existence of a nonnegative solution of problem (2.2)}

Consider the problem

$$
M_{0}^{\prime}=\inf _{u \in B\left(0, \rho_{1}\right)} I(u),
$$

where $\rho_{1}$ is defined in Lemma 3.4.

Remark 5.1 By the same arguments used in the proof of Lemma 4.1, we conclude that $-\infty<M_{0}^{\prime}<0$.

Theorem 5.2 Assume that hypotheses $\left(\overline{A_{1}}\right),\left(H_{1}\right),\left(H_{3}\right)$ and $(F)$ hold. Then there exists $V_{0} \in H^{1}\left(\mathbb{R}^{N}\right)$ such that $I\left(V_{0}\right)=M_{0}^{\prime}$. $V_{0}$ is a weak solution of problem 2.2.

Proof It is sufficient to replace Lemma 3.1 by Lemma 3.2 in the proof of Theorem 4.2.

\subsection{Existence of a second solution of problem (2.2)}

Lemma 5.3 Suppose that hypotheses $\left(\overline{A_{1}}\right),\left(H_{2}\right),\left(H_{3}\right), \quad(F)$ and $\left(N^{\mp}\right)$ hold. Then any $(P-S)$-sequence of $I$ is bounded in $H^{1}\left(\mathbb{R}^{N}\right)$.

Proof Let $\left(u_{n}\right)$ be a (PS)-sequence of $I$.

Case 1. We suppose that $a$ satisfies $\left(N^{+}\right)$. Arguing by contradiction, suppose that $\left\|u_{n}\right\|_{X} \rightarrow+\infty$. Then there exist $c>0$ and $d>0$ such that for $n$ large enough we have

$$
\left\|u_{n}^{\mp}\right\|_{X} \leq c\left\|u_{n}\right\|_{X}^{q}+d .
$$

Indeed, from $\left(\overline{A_{1}}\right)$ and for $n$ large enough we obtain

$$
\left\|u_{n}^{+}\right\|_{X} \geq\left\langle I^{\prime}\left(u_{n}\right), u_{n}^{+}\right\rangle \geq\left\|u_{n}^{+}\right\|_{X}^{2}-c\|a\|_{\frac{2}{1-q}}\left\|u_{n}\right\|_{X}^{q}\left\|u_{n}^{+}\right\|_{X}-c\|f\|_{2}\left\|u_{n}^{+}\right\|_{X},
$$

hence

$$
\left\|u_{n}^{+}\right\|_{X} \leq c\|a\|_{\frac{2}{1-q}}\left\|u_{n}\right\|_{X}^{q}+c\|f\|_{2}
$$


Similarly, from $\left(\overline{A_{1}}\right)$, we have

$$
-\left\|u_{n}^{-}\right\|_{X} \leq\left\langle I^{\prime}\left(u_{n}\right), u_{n}^{-}\right\rangle \leq-c\left\|u_{n}^{-}\right\|_{X}^{2}+c\|a\|_{\frac{2}{1-q}}\left\|u_{n}\right\|_{X}^{q}\left\|u_{n}^{-}\right\|_{X}+c\|f\|_{2}\left\|u_{n}^{-}\right\|_{X},
$$

for $n$ sufficiently large. Therefore

$$
\left\|u_{n}^{-}\right\|_{X} \leq c\left\|u_{n}\right\|_{X}^{q}+d .
$$

Using (5.2) we deduce

$$
\left\|u_{n}-u_{n}^{0}\right\|_{X}=\left\|u_{n}^{+}+u_{n}^{-}\right\|_{X} \leq c\left\|u_{n}\right\|_{X}^{q}+d
$$

and

$$
\lim _{n \rightarrow+\infty} \frac{\left\|u_{n}^{0}\right\|_{X}}{\left\|u_{n}\right\|_{X}}=1
$$

On the other hand, combining relations (5.2), (5.3), (5.4), $\overline{\left(A_{1}\right)}$ with the mean value theorem and the Hölder inequality, we obtain

$$
\begin{aligned}
I\left(u_{n}\right)= & \frac{1}{2}\left\|u_{n}^{+}\right\|_{X}^{2}-\frac{1}{2}\left\|u_{n}^{-}\right\|_{X}^{2}-\frac{1}{q+1} \int_{\mathbb{R}^{N}} a(x)\left[\left|u_{n}\right|^{q+1}-\left|u_{n}^{0}\right|^{q+1}\right] d x \\
& -\frac{1}{q+1} \int_{\mathbb{R}^{N}} a(x)\left|u_{n}^{0}\right|^{q+1} d x-\int_{\mathbb{R}^{N}} f(x) u_{n} d x \\
\leq & c\left\|u_{n}\right\|_{X}^{2 q}+d-\frac{1}{q+1} \int_{\mathbb{R}^{N}} a(x)\left[\left|u_{n}\right|^{q+1}-\left|u_{n}^{0}\right|^{q+1}\right] d x \\
& -\frac{1}{q+1} \int_{\mathbb{R}^{N}} a(x)\left|u_{n}^{0}\right|^{q+1} d x+c\|f\|_{2}\left\|u_{n}\right\|_{X}
\end{aligned}
$$

and

$$
\begin{aligned}
& \left|\int_{\mathbb{R}^{N}} a(x)\left[\left|u_{n}\right|^{q+1}-\left|u_{n}^{0}\right|^{q+1}\right] d x\right| \\
& \quad \leq c\|a\|_{\frac{2}{1-q}}\left(\left\|u_{n}\right\|_{X}+\left\|u_{n}^{0}\right\|_{X}\right)^{q}\left\|u_{n}-u_{n}^{0}\right\|_{X} \\
& \quad \leq c\left(\left\|u_{n}\right\|_{X}+\left\|u_{n}^{0}\right\|_{X}\right)^{q}\left(\left\|u_{n}\right\|_{X}^{q}+1\right) \\
& \quad \leq c\left(\left(\left\|u_{n}\right\|_{X}+\left\|u_{n}^{0}\right\|_{X}\right)^{q}+\left\|u_{n}\right\|_{X}^{2 q}+\left(\left\|u_{n}\right\|_{X}+\left\|u_{n}^{0}\right\|_{X}\right)^{2 q}\right) \\
& \quad \leq c\left(\left\|u_{n}\right\|_{X}^{2 q}+\left\|u_{n}\right\|_{X}^{q}\right)+c\left\|u_{n}^{0}\right\|_{X}^{2 q} \text {, for } n \text { sufficiently large. }
\end{aligned}
$$

Using (5.5) and (5.6) we deduce that

$$
\begin{aligned}
I\left(u_{n}\right) \leq c & \left(\left\|u_{n}\right\|_{X}^{2 q}+\left\|u_{n}^{0}\right\|_{X}^{2 q}+\left\|u_{n}\right\|_{X}^{q}\right) \\
& -\frac{1}{q+1} \int_{\mathbb{R}^{N}} a(x)\left|u_{n}^{0}\right|^{q+1} d x+c\|f\|_{2}\left\|u_{n}\right\|_{X}+d .
\end{aligned}
$$

As a consequence of $\left(N^{+}\right)$and Lemma 3.2, we obtain for all $0<d<q+1$

$$
\frac{1}{\left\|u_{n}^{0}\right\|_{X}^{d}} \int_{\mathbb{R}^{N}} a(x)\left|u_{n}^{0}\right|^{q+1} d x=\frac{\left\|u_{n}^{0}\right\|_{X}^{q+1}}{\left\|u_{n}^{0}\right\|_{X}^{d}} \int_{\mathbb{R}^{N}} a(x)\left(\frac{\left|u_{n}^{0}\right|}{\left\|u_{n}^{0}\right\|_{X}}\right)^{q+1} d x \rightarrow+\infty .
$$

Let us first assume that $\frac{1}{2}<q<1$. Relations (5.4) and (5.8) yield

$$
\begin{aligned}
I\left(u_{n}\right) \leq\left\|u_{n}^{0}\right\|_{X}^{2 q}\left[c\left(\frac{\left\|u_{n}\right\|_{X}^{2 q}}{\left\|u_{n}^{0}\right\|_{X}^{2 q}}+\frac{\left\|u_{n}\right\|_{X}^{q}}{\left\|u_{n}^{0}\right\|_{X}^{2 q}}+1\right)\right. \\
\left.\quad-\frac{1}{\left\|u_{n}^{0}\right\|_{X}^{2 q}} \int_{\mathbb{R}^{N}} a(x)\left|u_{n}^{0}\right|^{q+1} d x+c\|f\|_{2} \frac{\left\|u_{n}\right\|_{X}}{\left\|u_{n}^{0}\right\|_{X}^{2 q}}\right] \rightarrow-\infty .
\end{aligned}
$$


Therefore we reach contradiction and we conclude that the sequence $\left(u_{n}\right)$ is bounded in $H^{1}\left(\mathbb{R}^{N}\right)$.

Next, we assume that $0<q<\frac{1}{2}$. For $1<d<q+1$ and using (5.4) and (5.8), we obtain

$$
\begin{aligned}
I\left(u_{n}\right) \leq & c\left\|u_{n}^{0}\right\|_{X}^{d}\left(\frac{\left\|u_{n}\right\|_{X}^{2 q}}{\left\|u_{n}^{0}\right\|_{X}^{d}}+\frac{\left\|u_{n}^{0}\right\|_{X}^{2 q}}{\left\|u_{n}^{0}\right\|_{X}^{d}}+\frac{\left\|u_{n}\right\|_{X}^{q}}{\left\|u_{n}^{0}\right\|_{X}^{d}}\right) \\
& -\int_{\mathbb{R}^{N}} a(x)\left|u_{n}^{0}\right|^{q+1} d x+\|f\|_{2}\left\|u_{n}^{0}\right\|_{X}^{d} \frac{\left\|u_{n}\right\|_{X}}{\left\|u_{n}^{0}\right\|_{X}^{d}} \rightarrow-\infty .
\end{aligned}
$$

This yields to a contradiction and we conclude that the sequence $\left(u_{n}\right)$ is bounded in $H^{1}\left(\mathbb{R}^{N}\right)$.

Case 2. We suppose that $a$ satisfies $\left(N^{-}\right)$. We have

$$
\begin{aligned}
I\left(u_{n}\right) \geq & -\left\|u_{n}^{-}\right\|_{X}^{2}-\frac{1}{q+1} \int_{\mathbb{R}^{N}} a(x)\left[\left|u_{n}\right|^{q+1}-\left|u_{n}^{0}\right|^{q+1}\right] d x \\
& -\frac{1}{q+1} \int_{\mathbb{R}^{N}} a(x)\left|u_{n}^{0}\right|^{q+1} d x-\int_{\mathbb{R}^{N}} f(x) u_{n} d x \\
\geq & -c\left\|u_{n}\right\|_{X}^{2 q}-c-c\|a\|_{\frac{2}{1-q}}\left(\left\|u_{n}\right\|_{X}+\left\|u_{n}^{0}\right\|_{X}\right)^{q}\left\|u_{n}-u_{n}^{0}\right\|_{X} \\
& -\frac{1}{q+1} \int_{\mathbb{R}^{N}} a(x)\left|u_{n}^{0}\right|^{q+1} d x-c\|f\|_{2}\left\|u_{n}\right\|_{X}
\end{aligned}
$$

and

$$
\lim _{n \rightarrow+\infty} \frac{1}{\left\|u_{n}^{0}\right\|_{X}^{d}} \int_{\mathbb{R}^{N}} a(x)\left|u_{n}^{0}\right|^{q+1} d x=-\infty, \quad \forall 0<d<q+1 .
$$

Using the above inequalities and similar to Case 1 , we conclude that $\left(u_{n}\right)$ is bounded in $H^{1}\left(\mathbb{R}^{N}\right)$.

Theorem 5.4 We suppose that hypotheses $\left(\overline{A_{1}}\right),\left(H_{2}\right),\left(H_{3}\right)$ and $(F)$ hold. Then I satisfies the $(P S)$ condition.

Proof Let $\left(u_{n}\right) \subset H^{1}\left(\mathbb{R}^{N}\right)$ be a (PS)-sequence such that

$$
I\left(u_{n}\right) \rightarrow M_{1}^{\prime} \text { and } I^{\prime}\left(u_{n}\right) \rightarrow 0 \text { in }\left(H^{1}\left(\mathbb{R}^{N}\right)\right)^{\prime} .
$$

By Lemma 5.3, $\left(u_{n}\right)$ is bounded in $H^{1}\left(\mathbb{R}^{N}\right)$. Thus up to a subsequence, $u_{n} \rightarrow V_{1}$ in $H^{1}\left(\mathbb{R}^{N}\right)$, $u_{n} \rightarrow V_{1}$ in $L_{\text {loc }}^{m}\left(\mathbb{R}^{N}\right)$ for all $1 \leq m<2^{*}$ and $u_{n} \rightarrow V_{1}$ a.e. in $\mathbb{R}^{N}$.

By Del Pino's argument [23], it is sufficient to prove that for any $\epsilon>0$, there exist $R_{2}>0$ and $n_{0}>0$ such that

$$
\int_{|x| \geq R}\left(\left|\nabla u_{n}\right|^{2}+\left|u_{n}\right|^{2}\right) d x<\epsilon, \text { for all } R \geq R_{2} \text { and } n \geq n_{0} .
$$

Let $\phi_{R}$ be the cut-off function defined in (4.16). Since $I^{\prime}\left(u_{n}\right) \rightarrow 0$ as $n \rightarrow+\infty$ in $H^{1}\left(\mathbb{R}^{N}\right)^{\prime}$, for $\epsilon>0$, there exists $n_{0}>0$ such that

$$
\left|\left\langle I^{\prime}\left(u_{n}\right), \phi_{R} u_{n}\right)\right| \leq c\left\|I^{\prime}\left(u_{n}\right)\right\|\left\|u_{n}\right\|_{H} \leq \frac{\epsilon}{3}, \quad \forall n \geq n_{0}
$$

It follows that

$$
\begin{aligned}
& \int_{\mathbb{R}^{N}}\left(\left|\nabla u_{n}\right|^{2}+\left(V^{+}+\chi_{B\left(0, R_{0}\right)}\right)(x) u_{n}^{2}\right) \phi_{R} d x \\
& \leq \int_{\mathbb{R}^{N}}\left(V^{-}+\chi_{B\left(0, R_{0}\right)}\right)(x) \phi_{R} u_{n}^{2} d x+\int_{\mathbb{R}^{N}} a(x) \phi_{R}\left|u_{n}\right|^{q+1} d x \\
& \quad+\int_{\mathbb{R}^{N}} f(x) \phi_{R}(x) u_{n} d x-\int_{\mathbb{R}^{N}} u_{n} \nabla u_{n} \nabla \phi_{R} d x+\frac{\epsilon}{3} .
\end{aligned}
$$


Therefore

$$
\begin{aligned}
& \min (\beta, 1) \int_{\mathbb{R}^{N}}\left(\left|\nabla u_{n}\right|^{2}+u_{n}^{2}\right) \phi_{R} d x \\
& \quad \leq \int_{\mathbb{R}^{N}}\left(V^{-}+\chi_{B\left(0, R_{0}\right)}\right)(x) \phi_{R} u_{n}^{2} d x+\int_{\mathbb{R}^{N}} a(x) \phi_{R}\left|u_{n}\right|^{q+1} d x \\
& \quad+\int_{\mathbb{R}^{N}} f(x) \phi_{R}(x) u_{n} d x-\int_{\mathbb{R}^{N}} u_{n} \nabla u_{n} \nabla \phi_{R} d x+\frac{\epsilon}{3},
\end{aligned}
$$

From $(F),\left(H_{3}\right), \overline{\left(A_{1}\right)}$ and (4.16), there exists $R_{3}>0$ such that

$$
c\left(\left\|f \phi_{R}\right\|_{2}+\left\|\left(V^{-}+\chi_{B\left(0, R_{0}\right)}\right) \phi_{R}\right\|_{\frac{N}{2}}+\left\|a \phi_{R}\right\|_{\frac{2}{1-q}}+\frac{1}{R}\right) \leq \frac{\epsilon}{3} \text { for }|x| \geq R_{4} .
$$

The rest of the proof is similar to the second step of the proof of Lemma 4.4.

Using (4.2) in combination with hypothesis $\left(H_{1}\right)$, we construct $\varphi_{2}=t \phi \in B^{c}\left(0, \rho_{1}\right)$ for $t$ large enough, such that $I\left(\varphi_{2}\right)<0$ ( $\rho_{1}$ is fixed by Lemma 3.4). Consider the mini-max problem

$$
M_{0}^{\prime \prime}=\inf _{\gamma \in \Gamma_{0}^{\prime \prime}} \max _{t \in[0,1]} I(\gamma(t))
$$

where

$$
\Gamma_{0}^{\prime \prime}=\left\{\gamma \in C\left([0,1], H^{1}\left(\mathbb{R}^{N}\right)\right), \gamma(0)=0 \text { and } \gamma(1)=\varphi_{2}\right\} .
$$

Combining the mountain pass theorem [5] and Lemma 5.4, we deduce the existence of $W_{0} \in H^{1}\left(\mathbb{R}^{N}\right)$ such that $M_{0}^{\prime \prime}=I\left(W_{0}\right), I\left(W_{0}\right)>0$ and $W_{0}$ is a weak solution of problem (2.2).

\subsection{Concluding remarks}

The content of this paper is in relationship with the recent contributions of Bégout and Díaz [9-13] for the understanding of the nonlinear stationary Schrödinger equation. We expect that the methods developed by Díaz [24,25] in the case of an unbounded potential $V(x)$ of Hardy type can be applied to show that the nonnegative solutions of problem (2.2) established in Theorems 2.1 and 2.2 have a compact support (if $f(x)$ is also with compact support). This case corresponds to the abstract setting of infinite well type potentials.

Acknowledgements V. D. Rădulescu acknowledges the support through a grant of the Romanian Ministry of Research and Innovation, CNCS-UEFISCDI, project number PN-III-P4-ID-PCE-2016-0130, within PNCDI III. He was also supported by the Slovenian Research Agency Grants P1-0292, J1-8131, J1-7025, N1-0064, and N1-0083.

Open Access This article is distributed under the terms of the Creative Commons Attribution 4.0 International License (http://creativecommons.org/licenses/by/4.0/), which permits unrestricted use, distribution, and reproduction in any medium, provided you give appropriate credit to the original author(s) and the source, provide a link to the Creative Commons license, and indicate if changes were made.

\section{References}

1. Adachi, S., Tanaka, K.: Four positive solutions for the equation $-\Delta u+u=a(x) u^{p}+f(x)$ in $\mathbb{R}^{N}$. Calc. Var. Partial Differ. Equ. 11, 59-63 (2000)

2. Adachi, S., Tanaka, K.: Existence of positive solutions for a class of nonhomogeneous elliptic equation in $\mathbb{R}^{N}$. Nonlinear Anal. 48, 685-705 (2002)

3. Ahmad, S., Lazer, A.C., Paul, J.L.: Elementary critical point theory and perturbations of elliptic boundary value problem at resonance. Indiana Univ. Math. J. 25, 933-944 (1976) 
4. Ambrosetti, A., Badiale, M.: Homoclinics: poincaré-Melnikov type results via a variational approach. Ann. Inst. H. Poincaré Anal. Non Linéaire 15, 233-252 (1998)

5. Ambrosetti, A., Rabinowitz, P.H.: Dual variational methods in critical point theory and applications. J. Funct. Anal. 14, 349-383 (1973)

6. Ao, W., Wei, J.: Infinitely many positive solutions for nonlinear equations with non-symmetric potentials. Calc. Var. Partial Differ. Equ. 51, 761-798 (2014)

7. Bahrouni, A., Ounaies, H., Radulescu, V.: Infinitely many solutions for a class of sublinear Schrödinger equations with sign-changing potentials. Proc. R. Soc. Edinb. Sect. A 145A, 445-465 (2015)

8. Balabane, M., Dolbeaut, J., Ounaies, H.: Nodal solutions for a sublinear elliptic equation. Nonlinear Anal. 52, 219-237 (2003)

9. Bégout, P., Díaz, J.I.: On a nonlinear Schrödinger equation with a localizing effect. C.R. Acad. Sci. Paris Sér. I 342, 459-463 (2006)

10. Bégout, P., Díaz, J.I.: Localizing estimates of the support of solutions of some nonlinear Schrödinger equations: the stationary case. Ann. Inst. H. Poincaré Anal. Non Linéaire 29, 35-58 (2012)

11. Bégout, P., Díaz, J.I.: Self-similar solutions with compactly supported profile of some nonlinear Schrödinger equations. Electron. J. Differ. Equ. 2014(90), 1-15 (2014)

12. Bégout, P., Díaz, J.I.: A sharper energy method for the localization of the support to some stationary Schrödinger equations with a singular nonlinearity. Discrete Contin. Dyn. Syst. 34(9), 3371-3382 (2014)

13. Bégout, P., Díaz, J.I.: Existence of weak solutions to some stationary Schrödinger equations with singular nonlinearity. Rev. R. Acad. Cien. Serie A. Mat. (RACSAM) 109(1), 43-63 (2015)

14. Benrhouma, M.: Study of multiplicity and uniqueness of solution for a class of nonhomogeneous sublinear elliptic equations. Nonlinear Anal. 74, 2682-2694 (2011)

15. Benrhouma, M., Ounaies, H.: Existence of solutions for a perturbation sublinear elliptic equation in $\mathbb{R}^{N}$. NoDEA-Nonlinear Differ. Equ. Appl. 5, 647-662 (2010)

16. Benrhouma, M., Ounaies, H.: Existence and uniqueness of positive solution for a nonhomogeneous sublinear elliptic equation. J. Math. Anal. Appl. 385, 307-319 (2009)

17. Bolle, P.: On the Bolza problem. J. Differ. Equ. 152, 274-288 (1999)

18. Brezis, H., Kamin, S.: Sublinear elliptic equations in $\mathbb{R}^{N}$. Manuscr. Math. 74, 87-106 (1992)

19. Brown, K.J.: The Nehari manifold for a semilinear elliptic equation involving a sublinear term. Calc. Var. Partial Differ. Equ. 22, 483-493 (2004)

20. Cerami, G., Passaseo, D., Solimini, S.: Infinitely many positive solutions to some scalar field equations with non symmetric coefficients. Commun. Pure Appl. Math. 66, 372-413 (2013)

21. Chabrowski, J., Costa, D.G.: On a class of Schrödinger-type equations with indefinite weight functions. Commun. Partial Differ. Equ. 33, 1368-1394 (2008)

22. Costa, D.G., Tehrani, H.: Unbounded perturbations of resonant Schrödinger equations. In: Variational Methods: Open Problems, Recent Progress, and Numerical Algorithms, Contemp. Math., vol. 357, pp. 101-110. Amer. Math. Soc., Providence, RI (2004)

23. Del Pino, M., Felmer, P.: Local mountain passes for semilinear elliptic problems in unbounded domains. Calc. Var. Partial Differ. Equ. 4, 121-137 (1996)

24. Díaz, J.I.: On the ambiguous treatment of the Schrödinger equation for the infinite potential well and an alternative via flat solutions: the one-dimensional case. Interfaces Free Bound. 17, 333-351 (2015)

25. Díaz, J.I.: On the ambiguous treatment of the Schrödinger equation for the infinite potential well and an alternative via singular potentials: the multi-dimensional case. SeMA-J. (2017). https://doi.org/10.1007/ s40324-017-0115-3(published online 06 March 2017)

26. Floer, A., Weinstein, A.: Nonspreading wave packets for the cubic Schrödinger equation with a bounded potential. J. Funct. Anal. 69, 397-408 (1986)

27. Gamow, G.: Zur Quantentheorie des Atomkernes. Z. Phys. 51(204), 204-212 (1928)

28. Goubet, O., Hamraoui, E.: Blow-up of solutions to cubic nonlinear Schrödinger equations with defect: the radial case. Adv. Nonlinear Anal. 6(2), 183-197 (2017)

29. Joglekar, Y.N.: Particle in a box with $a$-function potential: strong and weak coupling limits. Am. J. Phys. 77, 734-736 (2009)

30. Kajikiya, R.: Multiple solutions of sublinear Lane-Emden elliptic equations. Calc. Var. Partial Differ. Equ. 26, 29-48 (2006)

31. Kato, T.: Remarks on holomorphic families of Schrödinger and Dirac operators. In: Knowles I., Lewis R. (eds.)Differential Equations, North-Holland Math. Stud., vol. 92, pp. 341-352, North-Holland, Amsterdam (1984)

32. Landesman, E.M., Lazer, A.C.: Nonlinear perturbations of linear elliptic boundary value problems at resonance. J. Math. Mech. 19, 609-623 (1970)

33. Li, T., Wu, T.F.: Existence of multiple positive solutions for nonhomogeneous elliptic problems in $\mathbb{R}^{N}$. Nonlinear Anal. 75, 5639-5692 (2012) 
34. Mawhin, J., Willem, M.: Critical points of convex perturbations of some indefinite quadratic forms and semilinear boundary value problems at resonance. Ann. Inst. H. Poincaré Anal. Non Linéaire 3, 431-453 (1986)

35. Mott, N.F.: An Outline of Wave Mechanics. Cambridge University Press, Cambridge (1930)

36. Oh, Y.G.: Existence of semi-classical bound states of nonlinear Schrödinger equations with potentials of the class $\left(V_{a}\right)$. Commu. Partial Differ. Equ. 13, 1499-1519 (1988)

37. Tehrani, H.: Existence results for an indefinite unbounded perturbation of a resonant Schrödinger equation. J. Differ. Equ. 236, 1-28 (2007)

38. Wang, Q., Zhang, Q.: Multiple solutions for a class of sublinear equations. J. Math. Anal. Appl. 389, 511-518 (2012) 